\title{
Association of HDL-Cholesterol Level and ApoB/ApoA Ratio with Coronary Artery Disease Severity and Type 2 Diabetes Mellitus
}

\author{
Philips Abraham ${ }^{1}$, Sachu Philip² \\ ${ }^{1}$ Department of Biochemistry, Al-Azhar Medical College, Thodupuzha, Kerala, India. \\ ${ }^{2}$ Department of Biochemistry, Vivekanandha Dental College for Women, Namakkal, Tamilnadu, India.
}

\section{ABSTRACT}

\section{BACKGROUND}

The ApoB/ApoA-I ratio reflects the balance between pro- and anti-atherogenic particles, all of which contain cholesterol. The ApoB/A ratio is valid in both genders of all ages and is independent of the lipid levels and phenotypes. This study has been designed to investigate the association between the ratio of apolipoprotein B (apo B)/ apolipoprotein A1 (apo A1) and HDL-C with severity of coronary artery disease in patients with and without type 2 diabetes mellitus.

\section{METHODS}

The present case control study was carried out in departments of Cardiology and Biochemistry, Vinayaka Missions Hospital, Salem. Fasting blood samples have been collected from 109 CAD patients and 71 control subjects. Serum lipids were measured enzymatically, ApoA-I and Apo B were analysed by immunoturbidimetric method. The study was approved by ethics committee and informed consent was obtained.

\section{RESULTS}

In our study, Apo A-1 level was significantly low (1.187 \pm 0.20$)$ and Apo B was found to be significantly high $(1.64 \pm 0.62)$ in CAD with diabetes subjects, when compared to CAD without diabetes. HDL level was found to be significantly lowered in CAD patients. A significant positive association was observed between ApoA-1 and lipid parameters in CAD subjects with HDL-C at $(\mathrm{p}=0.00)$.

\section{CONCLUSIONS}

Apo B/Apo A1 ratio may be a healthier forecaster than conventional lipid markers to explore the severity of CAD. This study suggests that instead of measurement of lipid profiles alone, a better indicator apo B, A-I and B/A-I ratio measurement, which in turn highlights the cholesterol balance, can reduce the delay in the recognition of CAD.

\section{KEY WORDS}

Apolipoprotein AI, Apolipoprotein B, Coronary Artery Disease (CAD), Diabetes Mellitus

\author{
Corresponding Author: \\ Dr. Sachu Philip, \\ Professor and HOD, \\ Department of Biochemistry, \\ Vivekanandha Dental College for \\ Women, Elayampalayam, \\ Tiruchengode, Namakkal Dist., \\ Tamilnadu, India. \\ E-mail: philipsachu1@gmail.com
}

DOI: $10.14260 /$ jemds/2020/403

Financial or Other Competing Interests: None.

How to Cite This Article:

Abraham P, Philip S. Association of $H D L-$ cholesterol level and $A P O B / A P O$ a ratio with coronary artery disease severity and type 2 diabetes mellitus. J. Evolution Med. Dent. Sci. 2020;9(25):1849-1853, DOI: 10.14260/jemds/2020/403

Submission 19-12-2019,

Peer Review 22-05-2020,

Acceptance 01-06-2020,

Published 22-06-2020.

\section{(i)}




\section{BACKGROUND}

Understanding of risk factors for coronary artery disease has been a major contribution to cardiology in the last 40 years. Hyperlipidaemia, a fundamental risk factor for coronary heart disease have been manifested with increased LDL level, decreased HDL and with elevated triglycerides (TGs). Many lines of evidence suggest that oxidation of low-density lipoprotein (LDL) plays an important role in the pathogenesis of atherosclerosis. Although LDL remains a primary target for CAD prevention according to guidelines of National Cholesterol Education Program Adult Treatment Panel (ATP) III -NCEP ATP III, all those who have been suffering from CAD were not having elevated LDL but were found to have derangement of other lipid fractions, generally a low HDL level. ${ }^{1}$ Type 2 diabetes mellitus (T2DM) has also been considered as the same risk factor as of CAD.

The clinical significance of lipid parameters and the lipid ratios are well established in several study literatures. The oxidation of LDL by HDL mediated transport mechanism involves binding and carrying away of oxidized and prooxidant molecules. But recent studies emphasis the role of on-high density lipoprotein cholesterol as novel predictor for coronary diseases. Apolipoprotein $\mathrm{B}$ has been hence considered as a better predictor of CAD than LDL-C. New data are hoarding in favour of apoproteins as more edifying risk factors for coronary artery diseases than conventional lipid parameters.

Apolipoproteins are the protein constituent of lipoproteins. ApoA-I is a protein synthesized mainly in the liver and to a lesser extent in the small intestine as a single 243 amino acid polypeptide chain and plays a crucial role in lipid metabolism. It is the prime apolipoprotein in HDL particles can 'pick up' excess cholesterol from peripheral cells and transfer it back to the liver in the HDL particles. The anti-inflammatory, antioxidant and anti-atherogenic properties of ApoA-I are documented. ${ }^{2}$ Moreover both Apo-A-I and paraoxonase enzyme mainly determines the antioxidant properties of HDL. ${ }^{3}$ HDL is responsible for reverse cholesterol transport and has Apolipoprotein A-l as the major protein 4 .

ApoB reveals the total atherogenic particles, but Apo B containing lipoproteins are responsible for lipid absorption and triglyceride homeostasis, sub endothelial retention of apo B-containing lipoproteins, a necessary initiating event of atherogenesis. ApoB produced in the liver also stabilizes and allows the transport of cholesterol and triglycerides in plasma VLDL, IDL, and sd-LDL (small dense Low density lipoprotein). Increased levels of apoB and LDL are risk factors for atherosclerosis.5,6

On the other hand, low levels of apo B may provide protection against atherosclerosis. The risk of disease progression may be more directly related to the total number of circulating atherogenic particles that enter the arterial wall, than to the concentration of cholesterol in LDL particles only, it has been suggested that apolipoprotein B could be a better predictor of risk than concentrations of cholesterol in the LDL fraction.

The ApoB/A ratio is a balance between pro and antiatherogenic particles and have been found to be valid in both genders of all ages and is independent on the lipid levels and phenotypes. ${ }^{7}$ Hence this study has been designed to find out the link between HDL-cholesterol and apoproteins with coronary artery disease severity.

\section{METHODS}

\section{Selection of Patients}

A case control study was done from a group of people who attended the department of cardiology and who were referred to the cardiology for angiography and were not on any medications, except for diabetes control medicine. They were selected for the study based on the coronary angiography report and degree of stenosis, and was carried out in department of Cardiology and Biochemistry, Vinayaka Missions Hospital, Salem. After getting a written consent the anthropometric parameters were collected. A detailed questionnaire; which include the medical details of study subjects as well as their family, age of onset of diabetes mellitus, CAD and its complications, hypertension, other chronic diseases, medications, patients socio-economic status.

\section{Grouping of Subjects}

1. 52 coronary artery disease patients without type 2 diabetes mellitus. (CAD without DM)

2. 57 coronary artery disease patients with type 2 diabetes mellitus. (CAD with DM).

3. 71 healthy population as normal control.

\section{Inclusion Criteria}

- Patients with established single, double and triple vessel CAD disease.

- Patients who had positive treadmill test for inducible ischemia and with history of essential hypertension.

- CAD patients with recent onset of diabetes mellitus and essential hypertension.

\section{Exclusion Criteria}

- Patients excluded were those diagnosed to have coronary artery disease with atrial fibrillation or pacemaker and history of congestive heart failure.

- Patients with history of stroke, with transient ischemia or carotid surgery

- With history of coronary artery bypass graft surgery.

- Patients with transluminal coronary angioplasty and peripheral vascular surgery.

The serum glucose (FBG) was estimated by enzymatic method, lipid parameters except LDL by direct gold standard enzymatic colorimetric method. LDL-C and VLDL-C measured using Friedewald formula. ${ }^{8,9}$ Apo Aland Apo B were measured in by immunoturbidimetric method. ${ }^{8}$

\section{Baseline Examination}

Standard 12-lead ECG has been obtained and was coded using the Minnesota Code (MC). ${ }^{10}$ ECGs with abnormal QS pattern (defined as Minnesota codes 4.1-2), T-wave inversion (codes 5.1-2; T wave inversion $1.0 \mathrm{~mm}$ ). 
Grading of CAD Patients Based on Coronary Angiography The severity of coronary atherosclerosis were assessed by Judkins technique based on the number of vessels involved. Coronary artery disease was confirmed when they had more than $50 \%$ stenosis in at least one coronary artery segment. ${ }^{11}$ The subjects were graded as Grade I (mild) with severity of disease as $<50 \%$ stenosis, with single vessel involved and abnormal QS pattern with 2 leads and $\mathrm{T}$ wave inversion 2 leads. Grade II (moderate) with severity of disease 50-74\% stenosis, with double vessel involved and abnormal QS pattern with 3-5 leads and T wave inversion 3-5 leads. Grade III (severe) with severity of disease $75-100 \%$ stenosis, with triple vessel involvement and abnormal $Q S$ pattern with $>5$ leads and $\mathrm{T}$ wave inversion $>5$ leads.

\section{Statistical Analysis}

All data expressed as means \pm SD. One way analysis of variance (ANOVA) has been carried out. Results are significant at $p$ values $\leq 0.05$.

\section{RESULTS}

Our study population were in the age of 40 to 75 years. $65 \%$ were males and $35 \%$ were female among the normal study subjects. While $88 \%$ were males in CAD without diabetes and $82 \%$ in CAD with diabetes, and $12 \%$ were female in CAD without diabetes $18 \%$ in CAD with diabetic complications. The mean age of onset of symptoms of CAD in the group with diabetes was $52 \pm 4.5$ when compared to $59 \pm 6.4$ in the other group; while the mean extent of diabetes was $6.2 \pm 2.5$. SBP and DBP does not show any significant changes among the study group. Among the subjects BMI was found to be significant in the disease subjects with diabetes subjects when compared to CAD without diabetes and normal group (with $\mathrm{p}<0.001$ ).

\section{Demographic Details of Study Subjects}

The incidence of CAD was high in patients of the age group 51$60(77 \%)$ in CAD with type 2 DM patients when compared to CAD without DM (46\%). BMI was found to be in the range of $18.5-24.9 \mathrm{~kg} / \mathrm{m}^{2}$ in $2 \%$ of CAD without diabetes, when compared to $11 \%$ in CAD with diabetes. BMI was in the range of $25-30 \mathrm{~kg} / \mathrm{m}^{2}$ for $54 \%$ of CAD without diabetes compared to $21 \%$ in CAD with diabetes, also $44 \%$ of CAD without diabetes had BMI value $>30 \mathrm{~kg} / \mathrm{m}^{2}$ when compared to $68 \%$ in CAD with diabetes Insulin resistance has been found to be $1.7 \pm 0.33$ in normal subjects, $1.8 \pm 0.445$ in CAD without $\mathrm{DM}$ and $9.7 \pm 1.1$ in CAD with DM respectively. Insulin resistance has been found to be significantly elevated in CAD with diabetes group when compared to other two groups; which might be the principal defect in $>90 \%$ of coronary artery disease patients with diabetes.

\begin{tabular}{|c|c|c|c|}
\hline \multirow{2}{*}{ Severity of CAD } & \multicolumn{2}{|c|}{ CAD Subjects } & \multirow{2}{*}{ Total } \\
\hline & CAD without DM & CAD with DM & \\
\hline Grade I (mild) & $24(46 \%)$ & $9(16 \%)$ & $33(62 \%)$ \\
\hline Grade II (moderate) & $15(29 \%)$ & $12(21 \%)$ & $27(50 \%)$ \\
\hline Grade III (severe) & $13(25 \%)$ & $36(63 \%)$ & $49(88 \%)$ \\
\hline \multicolumn{4}{|c|}{ Table I. Severity of CAD among the Study Subjects } \\
\hline
\end{tabular}

Analysis was done by Chi square test. The severity was significantly high in CAD with diabetes. (Table 2). A significantly high percentage of multi-vessel atherosclerosis was observed in coronary artery disease subjects when compared subjects without diabetes.

\begin{tabular}{|c|c|c|c|c|}
\hline $\begin{array}{c}\text { Lipid } \\
\text { Parameters }\end{array}$ & $\begin{array}{l}\text { CAD with DM } \\
(\mathrm{N}=52)\end{array}$ & $\begin{array}{l}\text { CAD without } \\
\text { DM }(N=57)\end{array}$ & $\begin{array}{l}\text { Control } \\
(\mathrm{N}=71)\end{array}$ & $\mathbf{P}$ \\
\hline ApoA-1 ( g\%) & $1.187 \pm 0.2051$ & $0.873 \pm 0.007 \mathrm{a}, \mathrm{b}$ & $1.39 \pm 0.31$ & 0.157 \\
\hline Аро B (g/\%) & $1.64 \pm 0.62^{\mathrm{a}}$ & $1.66 \pm 1.10^{\mathrm{a}, \mathrm{b}}$ & $0.79 \pm 0.12$ & 0.01 \\
\hline ApoB/Apo A-l ratio & $2.04 \pm 0.84$ & $2.069 \pm 1.23^{a}$ & $0.59 \pm 0.173$ & 0.0145 \\
\hline $\begin{array}{c}\text { Total serum } \\
\text { cholesterol }(\mathrm{mg} \%)\end{array}$ & $182.2 \pm 8.485^{\mathrm{a}}$ & $197.49 \pm 7.77^{a}$ & $169 \pm 8.0$ & 7.645 \\
\hline $\begin{array}{c}\text { Serum Triglycerides } \\
\text { (mg\%) }\end{array}$ & $131.6 \pm 6.4$ & $157.5 \pm 8.5^{\mathrm{a}, \mathrm{b}}$ & $\mathrm{I} 21.35 \pm 11.28$ & 12.95 \\
\hline HDL-C (mg\%) & $38.4 \pm 2.07 \mathrm{a}$ & $35.05 \pm 1.44^{\mathrm{a}, \mathrm{b}}$ & $41.48 \pm 5.59$ & 1.675 \\
\hline LDL -C (mg\%) & $129.50 \pm 8.8^{\mathrm{a}}$ & $144.25 \pm 8.2^{\mathrm{a}, \mathrm{b}}$ & $104.48 \pm 33.5$ & 7.375 \\
\hline VLDL (rng\%) & $26.3 \pm 13.01$ & $31.5 \pm 9.617^{a}$ & $24.27 \pm 2.26$ & 2.6 \\
\hline \multicolumn{5}{|c|}{$\begin{array}{c}\text { Table 2. Apolipoprotein and Lipid Parameters } \\
\text { among the Study Subjects }\end{array}$} \\
\hline & ant at $\mathrm{p}<0.05, \mathrm{~b}$ : $\mathrm{si}$ & ificant at $\mathrm{p}<0.05$ & & \\
\hline
\end{tabular}

ApoA-1, ApoB, and ApoB/A Ratio in Study Subjects The ratio of Apo B/A1 was found to be very significant at $\mathrm{p}<0.05$ in CAD with diabetic subjects and also a significantly low level Apo A-1 $(\mathrm{p}<0.001)$ in the diabetic subjects when compared to CAD without diabetes. This might be due to the presence of high level of Apo E which causes the catabolism of Apo A-1 and HDL. A correlation analysis of ApoA-1, lipid profile and HDL in CAD without diabetic subjects, showed a significant positive association with HDL-C $(\mathrm{r}, .755, \mathrm{p}=.000)$. ApoA-1 also showed positive association with HDL-C $(r, .415, p=.002)$ In CAD without diabetes.

\begin{tabular}{|c|c|c|c|c|}
\hline \multirow{8}{*}{$\begin{array}{c}\mathrm{HDL} \\
(\mathrm{mg} / \mathrm{dL})\end{array}$} & \multicolumn{4}{|c|}{ Distribution of CAD Subjects (\%) } \\
\hline & & \multicolumn{2}{|c|}{ CAD without DM } & CAD with DM \\
\hline & $<40.0 \mathrm{mg} / \mathrm{dL}$ & \multirow{2}{*}{\multicolumn{2}{|c|}{$\begin{array}{l}56 \\
44\end{array}$}} & 77 \\
\hline & $40.0-60.0 \mathrm{mg} / \mathrm{dL}$ & & & 23 \\
\hline & \multicolumn{4}{|c|}{ Percentage of stenosis (\%) } \\
\hline & & Grade I & Grade II & Grade III \\
\hline & $<40.0 \mathrm{mg} / \mathrm{dL}$ & $32 \%$ & $25 \%$ & $16 \%$ \\
\hline & $40.0-60.0 \mathrm{mg} / \mathrm{dL}$ & $19 \%$ & $14 \%$ & $3 \%$ \\
\hline & Table III. HD & vel in $C$ & D Subjects & \\
\hline
\end{tabular}

The HDL-C level was found to be not significant between the CAD with DM and CAD without DM. (Table 3). ApoB/A ratio has been found to be significant at $\mathrm{p}<0.05$ while, the level of Apo A1 was significantly low $(\mathrm{p}<0.001)$ in CAD with diabetic subjects, when compared to CAD subjects without diabetes and controls. High level of Apo- E which effects the catabolism of Apo A-1 and HDL; might be the reason for the significant increase in the ratio in CAD with diabetic subjects. Correlation analysis has been done, between ApoA-1 and lipid profile in CAD without DM and had shown a signifying positive correlation with HDL-C ( $\mathrm{r}, 755, \mathrm{p}=.000)$. ApoA-1 also showed significant positive association with $\operatorname{HDL}(\mathrm{r}, 415, \mathrm{p}=.002)$ in CAD without diabetic subjects.

\section{DISCUSSION}

The present study has been aimed to find out the relationship between HDL-cholesterol and apolipoprotein with coronary artery severity. It has been found that a delayed recognition of CAD was noticed in diabetic subjects in our study. In diabetic patients, the signs of cardiac ischemia are often concealed and 
hence in the preliminary stages; the pathological events are not been recognized. The frequency of grade III severity was comparatively high and percentage of high mortality in diabetic patients with CAD may be due to this fact. Various epidemiological studies also showed a similar result. ${ }^{12}$

The cholesterol level was significantly high in CAD patients. The association of cholesterol with coronary artery disease was demonstrated by the Framingham study, and the seven countries study also demonstrated the elevated plasma cholesterol levels with increased incidence of CHD. ${ }^{13}$ As the cholesterol concentration increases, lipoproteins with apo B infiltrate the arterial wall, altered to a toxic state and initiate an inflammatory processes. Increased infiltration may be caused by endothelial denudation. The development of atheroma are often covered by an intact endothelial layer with stages of lesion progressions, retention of lipoproteins, formation of fatty streak and leads to advanced lesions. ${ }^{14}$ Sometimes endothelial injuries cause increased permeability. ${ }^{14}$

The individuals with higher cholesterol levels were found to be more prone for developing CHD, but it has been established for those with no other risk factors as well as those with added risk. The other major risk factors may exert an adverse effects via their effects on cholesterol metabolism. Hence cholesterol alone can be a poor predictor and hence; in our study cholesterol level does not show significant change with severity.

Triglycerides have confrontational effect on impaired endothelial functioning. The involvement of hyper triglyceridaemia in progression of coronary artery disease at various level might be the etiological factor for positive correlation of TG and severity of the disease. ${ }^{15}$ The prominence of LDL-C in the progression of coronary artery disorder has been well established. The proteoglycans produced by the smooth muscle cells of the arterial wall interact with apoB of LDL cholesterol, thereby increasing the retention of LDL. Significant elevation of LDL level in CAD with diabetes subjects has been found in our study. Any LDL-C reduction has been found to be associated with less atherosclerosis progression and decreased rate of revascularization. The size of LDL particles mainly influence the entry of LDL into the endothelial tissues, and leads to early $r$ dys functioning of vascular tissues expressed of impaired endothelial retort in patients with diabetes. This LDL fraction might be independent of other variables, including lipid levels, BMI, blood pressure and severity. Hence the absolute LDL- concentration directly does not reflect the number of increased; atherogenic particles and found to be misleading. Steinberg et al suggested that oxidized LDL promotes the immune and inflammatory reactions that progress to atherosclerosis. No positive correlation between LDL cholesterol with Insulin resistance and severity of CAD may be due to this. ${ }^{16}$

Our results showed that HDL-C level was significantly hand down in CAD patients compared to normal. ${ }^{17}$ The reverse association between HDL-C levels and atherosclerosis are well proven by clinical studies. HDL-C reduces the risk of CAD by reducing the progression of atherogenesis. HDL-C mainly causes the reverse cholesterol transport thereby interacts with foam cells to remove free cholesterol from the peripheral tissues and its excretion through liver. Other major protective role HDL-C are potentiation of endothelial function and repair, anti-inflammatory property, antithrombotic, and antioxidant activity. HDL It plays a key role in the metabolism of LDL and VLDL. Functions of HDL cholesterol vary according to its particle size and its apo protein Content HDL-C particle size increases with more cholesterol content, but the transport of cholesterol into the liver gets decreased and results in the progression of atherosclerosis. HDL A-I is more effective than HDL A-I: A-II in promoting cholesterol efflux, which is consistent with the at hero protective effect of apo A-I on LCAT. 18

Reduced HDL cholesterol levels found in diabetic subjects with CAD in this study may be due to the high Apo E-containing triglyceride-rich lipoproteins. Also, the elevation of circulating apo E-containing triglyceride-rich lipoproteins could lead to increased transfer of apo E to LpAI/AII (Lipoprotein AI/AII). ${ }^{19}$

The incidence of coronary artery diseases and relation of HDL related parameters including HDL-C, and its apoproteins A-I, and A-II are well established by previous studies. The 'HDL and its lipoproteins were related to the risk for CAD, but HDL and apo A-I were the promising forecaster. Henceforth no significant positive correlation was observed between HDL and severity in our study.

The ApoA-I of HDL particles should be assessed for cardiovascular risk in humans. HDL subpopulations diverge in their ability to protect against CHD. Briteet al related HDL subpopulations to $\mathrm{CAD}$, found that both the severity and the rateofprogressionofatherogeniclesionscorrelatesignificantlya ndinverselywiththeconcentration of HDL subfractions. ${ }^{20}$

Apolipoprotein are more under genetic control than lipid components and hence overwhelms the lipids. Hence they illustrate the precise number of lipoprotein particles. Apo A-1 is a better predictor than HDL. It is the major structural protein of HDL (70\%) and it plays major role in HDL metabolism. Furthermore, apo A-I is the ligand for the ATP-binding cassette $(\mathrm{ABC})$ protein, $\mathrm{ABCA} 1$ and is involved in the removal of excess cholesterol in peripheral cells. Apo A also increases the hydrolysis of triglycerides and so its deficiency has been found to be associated with atherosclerosis.

Based on previous literature support and recent studies have established that the predominant risk factors for cardiovascular (CV) diseases are increased IDL fraction and VLDL fractions. Apo B is a large amphipathic protein and is found to be present in non HDL lipoproteins; VLDL, IDL and LDL. ApoB is vital for the intracellular aggregation in liver for VLDL and in RBC for chylomicrons. Apo B apolipoprotein and non HDL-C lipoproteins are indicator of total number of atherogenic molecules. In our study Apo B was significantly high in CAD with diabetes subjects when compared to the other CAD group and healthy group.

Atherosclerosis is initiated by the subendothelial withholding of apoB-containing lipoproteins mainly LDL, LDL remnants, and Lipoprotein (a). ApoB containing LDL can coerce the development of atherosclerosis even if the other risk factors are masked.

Hence apo B can be a better analyst of CV risk than LDL-c, VLDL and chylomicrons. Apo B independently predicts endothelial vasodilator property, increased carotid thickening and arterial stiffness. All these evidences wrap up the fact that an excess of apo B- particles always denote proatherogenic phenomenon and promotes the severity. Hence a ratio of ApoB/ ApoA-1 was considered to get an accurate image of atherogenic and antiatherogenic progression by apo B and apo A-1 molecules. The reason for improved predictive effect of 
these ratio might be due to the fact that it reflects and integrates the Cholesterol level between potentially atherogenic lipoprotein particles (ApoB) in relation to all anti atherogenic particles. (ApoA-1) Apo B/Apo A1 ratio affirms to be better predictor of CAD; than the lipid profile parameters individually or the ratio of other lipoproteins. The methodological errors in the assessment of the apolipoprotein are negligible since they are estimated by standardized techniques than the assessment of conventional lipid parameters; where LDL is estimated by Friedewald formula.. ${ }^{21}$

Significantly higher values of ApoB/A-1 has been found in diseased subjects, irrespective of their diabetes status. The ratio and severity was not significant in non-diabetic subjects at $r=1.000$ with $p=.809$ it was significant at $p<.05$ in subjects with diabetic at; $r=.547$ and $p=.034$. The relation of Apo B and ApoA-1; analysed by the Odds ratio and was 0.48 for CAD subjects without diabetes while for CAD with diabetes it seems to be 4.5 ; (95\% CI: 1.122 - 18.31). AMORIS study also affirms that the ratio may be better predictor of CAD risk than LDL-C, are more strongly concomitant with coronary diseases.

\section{CONCLUSIONS}

The management of diabetes mellitus has improved to a great extent, but distinctive symptoms of cardiac manifestations are frequently concealed in people with diabetes. Routine assessment of lipid profile could not fully explain the occurrence of CAD. Hence, this study suggests that instead of measuring lipid profiles alone, better indicators would be apo B, A-I and B/A-I ratio, which in turn highlights the cholesterol balance and can reduce the delay in the recognition of CAD.

\section{REFERENCES}

[1] Robinson JG, Stone NJ. The 2013 ACC/AHA guideline on the treatment of blood cholesterol to reduce atherosclerotic cardiovascular disease risk: a new paradigm supported by more evidence. Eur Heart J 2015;36(31):2110-8.

[2] Brites F, Martin M, Guillas I, et al. Antioxidative activity of high-density lipoprotein (HDL): mechanistic insights into potential clinical benefit. BBA Clin 2017;8:66-77.

[3] Tomás M, Latorre G, Sentí $M$, et al. The antioxidant function of high density lipoproteins: a new paradigm in atherosclerosis. Rev Esp Cardiol 2004;57(6):557-69.

[4] Woudberg NJ, Pedretti S, Lecour S, et al. Pharmacological intervention to modulate HDL: what do we target? Front Pharmacol 2018;8:989.

[5] Philip S, Abraham P, Sheriff DS. Apo B/Apo A-I ratio A better predictor of coronary artery disease in patients with or without type ii diabetes mellitus. International Journal of Applied Biology and Pharmaceutical Technology 2011;2(3):153-8.

[6] Sathe CA, Chogle SA, Bharadwaj D. Apo B/Apo A1 ratio: a risk marker in patients with cardiovascular disease. Int J Res Med 2017;6(2):20-3.
[7] Jun JE, Choi YJ, Lee YH, et al. ApoB/ApoA-I ratio is independently associated with carotid atherosclerosis in type 2 diabetes mellitus with well-controlled LDL cholesterol levels. Korean J Intern Med 2018;33(1):13847.

[8] Sheriff DS, Sachu P, Elshaari FA. HDL, apo B/apo A1 ratio, diabetes mellitus and cardiovascular disease. In: Masuo K, ed. Type 2 diabetes. Intech Open 2013.

[9] Kannan S, Mahadevan S, Ramji B, et al. LDL-cholesterol: friedewald calculated versus direct measurement-study from a large Indian laboratory database. Indian J Endocrinol Metabol 2014;18(4):502-4.

[10] Mahmoodzadeh S, Moazenzadeh M, Rashidinejad H, et al. Diagnostic performance of electrocardiography in the assessment of significant coronary artery disease and its anatomical size in comparison with coronary angiography. J Res Med Sci 2011;16(6):750-5.

[11] Zhou FF, Liu YH, Ge PC, et al. Coronary artery diameter is inversely associated with the severity of coronary lesions in patients undergoing coronary angiography. Cellular Physiology and Biochemistry 2017;43(3):1247-57.

[12] Philip S, Abraham P. Relation of insulin resistance and BMI with severity of coronary artery disease in patients with and without diabetes mellitus. Int J Pharm Sci Res 2018;9(11):4794-9.

[13] Agrawal N, Corradi PF, Gumaste N, et al. Triglyceride treatment in the age of cholesterol reduction. Prog Cardiovasc Dis 2016;59(2):107-18.

[14] BeLue R, Okoror TA, Iwelunmor J, et al. An overview of cardiovascular risk factor burden in sub-Saharan African countries: a socio-cultural perspective. Global Health 2009;5:10.

[15] Vergès B. Pathophysiology of diabetic dyslipidaemia: where are we? Diabetologia 2015;58(5):886-99.

[16] Karlson BW, Palmer MK, Nicholls SJ, et al. A VOYAGER meta-analysis of the impact of statin therapy on lowdensity lipoprotein cholesterol and triglyceride levels in patients with hypertriglyceridemia. Am J Cardiol 2016;117(9):1444-8.

[17] Estrada-Luna D, Ortiz-Rodriguez M, Medina-Briseño L, et al. Current therapies focused on high-density lipoproteins associated with cardiovascular disease. Molecules 2018;23(11):2730.

[18] Jenkins AJ, Yu J, Alaupovic P, et al. Apolipoprotein-defined lipoproteins and apolipoproteins: associations with abnormal albuminuria in type 1 diabetes in the diabetes control and complications trial/epidemiology of diabetes interventions and complications cohort. J Diabetes Complications 2013;27(5):447-53.

[19] Hafiane A, Genest J. HDL, atherosclerosis, and emerging therapies. Cholesterol 2013;2013:1-18.

[20] Brites F, Martin M, Guillas I, et al. Antioxidative activity of high-density lipoprotein (HDL): mechanistic insights into potential clinical benefit. BBA Clin 2017;8:66-77.

[21] Kaneva AM, Potolitsyna NN, Bojko ER, et al. The apolipoprotein B/apolipoprotein A-I ratio as a potential marker of plasma atherogenicity. Dis Markers 2015;2015:1-7. 